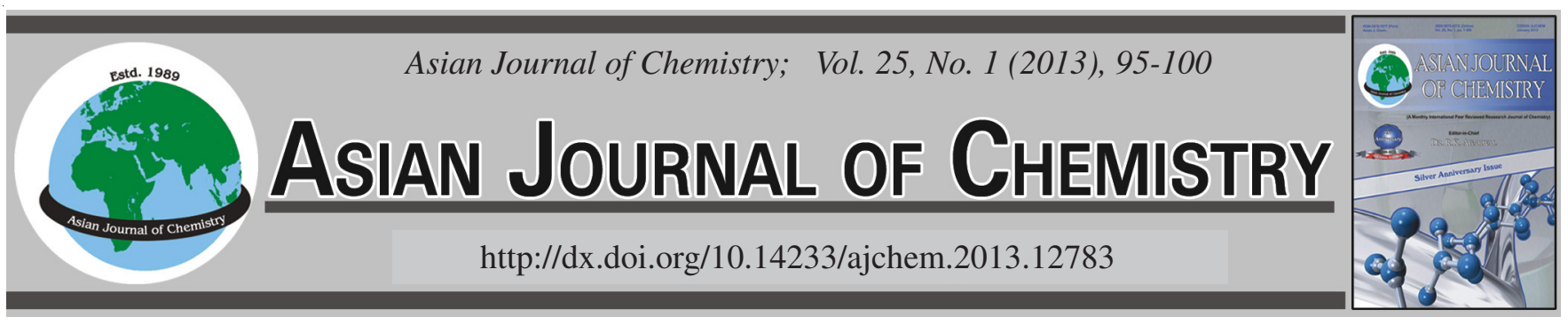

\title{
Virtual Screening of SdiA Inhibitors from Melia dubia to Curtail Uropathogenic E. coli Quorum Sensing
}

\author{
Vinothkannan Ravichandiran, S. Karthi, R. Shrimathi and S. Adline Princy*
}

Quorum Sensing \& Peptidomimetics Laboratory, Sastra's Hub for Research and Innovation, SASTRA University, Thirumalaisamudram, Thanjavur613 401, India

*Corresponding author: Tel: +91 4362 264101-108; E-mail: adlineprinzy @ biotech.sastra.edu

(Received: 26 September 2011;

Accepted: 9 July 2012)

AJC-11827

\begin{abstract}
Quorum sensing has been implicated to control the biofilm formation and virulence factors among many bacteria. SdiA, a transcriptional regulator of uropathogenic $E$. coli quorum sensing system that controls virulence arsenal. Natural products offer unlimited opportunities for new drug leads because of the utmost availability of chemical diversity. Here, the potentiality of five different extracts of Melia dubia root for quorum sensing inhibitory activity were investigated against uropathogenic Escherichia coli (UPEC). The assays such as cell density, swarming motility, protein, protease, hemolysis, hemagglutination, hydrophobicity and biofilm inhibition were performed. The biofilm, hemolysis, swarming motility were found to be inhibited by $60.6,14.45$ and $22.64 \%$, respectively when the media was supplemented with $30 \mathrm{mg} / \mathrm{mL}$ of ethanolic extract and other parameters were also found decreased. The GC-MS spectrum of ethanolic extract showed an array of 38 structurally unlinked compounds with the natural ligands. From this docking studies, it is observed that the compound 7 and $\mathbf{2 7}$ are having better Glide Score of $11.5,11.2$ respectively than the native ligand $\mathrm{C}_{8} \mathrm{HSL}$ (9.4). Compound $\mathbf{7}$ forms three hydrogen strong bond interactions with the amino acid residues TRP67, ASP80 and SER43 and compound 27 forms two hydrogen bond interactions with TRP67 and SER43. The interacting amino acids were almost same in C 7, C 27 and $\mathrm{C}_{8} \mathrm{HSL}$. The in vitro and in silico docking analysis and drug likeness prediction data showed fairly good correlation, suggesting that the ethanolic extract have the potency to attenuate the quorum sensing of uropathogenic E. coli. Further studies by in vitro and in vivo strategies are mandatory to find out the quorum quenching effect of the ligands.
\end{abstract}

Key Words: Quorum sensing, Quorum quenching, Melia dubia, Uropathogenic E. coli, SdiA.

\section{INTRODUCTION}

Urinary tract infection (UTI) is a common bacterial infection affecting any/all parts of urinary tract. Its prevalence is more in female than in male, studies rate the infection to occur by 1 in 3 women of world's population ${ }^{1}$. The reason is that in women the shorter length of urethra paves way for easy invasion of bacteria to the urinary tract than in male. Urinary tract infection is caused mainly by two bacterium namely, Escherichia coli (80-85\%) and Staphylococcus saprophyticus $(5-10 \%)^{2}$. The pathogenic form of $E$. coli causing urinary tract infection and that resisting the action of multiple drugs and antibiotics due to its biofilm formation are termed as uropathogenic E. coli (UPEC). This pathogenic form of $E$. coli is responsible for food toxicity, production of lethal toxins in small intestines etc. ${ }^{3}, E$. coli is one of the normal micro floras of human intestine and causes problem only when it migrates from the intestine to urinary bladder.

The E. coli that has migrated gets into the kidney or urinary bladder and multiplies in urine. They use quorum sensing (QS) mechanism to co-ordinate with their cells. Quorum sensing mechanism is a bacterial communication system through which the bacterial cells communicate in the form of chemical signaling molecules called autoinducers (AI) or quoromones ${ }^{4}$. Knowing the quorum sensing of a bacterium are very important as it orchestrate the network using which certain characters like virulence, bioluminescence and resistance towards drugs are expressed in a bacterium. Several gram negative bacteria produce AHLs, diketopiperazines as their quoromones $^{5}$. E. coli produces AI-2 along with Indole as quoromones in the presence trytophanase enzyme ${ }^{6}$. This special property of $E$. coli helps it to find the interference of any other bacteria in their surroundings. E. coli apart from quoromones consists of other proteins which play important role in regulating the expression of genes.

A protein, SdiA is the main reason behind the altered or regulated expression of any gene in E. coli. The molecular weight of this protein product is found to be $29 \mathrm{kDa}^{7}$. SdiA proportionally up regulates the virulence character ${ }^{9}$ in E. coli. 
This protein is the homologue of LuxR and its activity is up regulated through $\mathrm{N}$-aryl homoserine lactones. Thus the SdiA can be used to detect any AHLs synthesized by other bacteria. The SdiA in the absence of AHLs exists as insoluble inclusion bodies but once when it gets bound with AHLs it exhibits a folding switch model that will de-populate the other microbes ${ }^{7}$. The main role of SdiA is that it regulates the AcrA pump that is responsible for multi drug resistance (MDR) property of E. coli, thus the over expression of SdiA makes E. coli a MDR$E$. coli. Currently the E. coli have gained resistance to even modern drugs in the market, certain common drugs like mitomycin that are prescribed for $E$. coli - infected treatments are now not efficient enough to use ${ }^{8}$.

In order to attenuate $E$. coli and many other bacteria that are becoming multi drug resistant the need for the use of signal molecule based drugs to ameliorate bacterial pathogenecity instead of growth inhibition is encouraged and the inhibitors that down regulated the quorum sensing of a bacteria are called as quorum sensing inhibitors (QSI) ${ }^{9}$. Few quorum sensing inhibitor like garlic extracts,4-nitropyridine-N-oxide also proved effective against bacterial infections ${ }^{10}$. These drugs specifically target the quorum sensing of the bacteria in certain manner which do not pose pressure on the bacteria to self mutate them ${ }^{11}$. Hence several herbal plants are been taken under study to develop new quorum sensing inhibitors.

India, a country rich in flora and fauna bears the credit of using traditional herbal methods to cure some common disorders and infection. One of the bacterial infections that are often treated using herbal plants is urinary tract infection. Extracts from the plant Melia dubia from the family Melicacea more commonly called as "Malai Vembu" is prevalent in the usage for urinary tract infection. This plant had been used in 19th century for worm troubles and to encourage conception in women ${ }^{12}$. This plant was also grown with sugar plant and wheat to increase the yield. This plant is found to have several antidiabetic ${ }^{13}$, antiviral ${ }^{14}$, antifeedant ${ }^{15}$ properties.

In this investigation we aimed to screen the SdiA inhibitors from $M$. dubia root extracts against uropathogenic E. coli quorum sensing and to predict the drug likeness of the inhibitors.

\section{EXPERIMENTAL}

The bacterial strain of Escherichia coli UPEC/QSPL/S4 which showed maximum resistance to antibiotics like ampicillin, ciprofloxacin, levofloxin, nitrofurontoin and trimethoprim was isolated from patients in K.A.P. Vishwanathan Government Medical College, Trichy between September to December 2009 suffering from Urinary tract infection which caused by uropathogenic $E$. coli. This isolated strain was then cultured using LB (Luria Bertani) broth at a temperature of $37^{\circ} \mathrm{C}$ for ca. $24 \mathrm{~h}$ and this culture was utilized for further studies.

Extraction of plant material: Melia dubia, of the family Meliaceae used for the experiments were collected from in and around Kumbakonum, Tamilnadu between August to November 2009. This voucher herbarium specimen TUH 285 submitted to the Department of Environmental and Herbal Science, Thanjavur, TamilNadu, India was authentified by Dr. M. Jagadeesan, the head of the department. Cold Percolation method was employed for the extraction of the plant material from the powdered root of the Melia dubia ${ }^{16}$.

The extraction was done at room temperature $\left(25 \pm 1^{\circ} \mathrm{C}\right)$ using five different solvents like water, ethanol $(70 \%)$, methanol (70 \%), petroleum ether (70 \%) and hexane (70\%) with 1:10 $\mathrm{w} / \mathrm{v}$. The extracted samples were agitated at frequent intervals and a muslin cloth was used to filter the supernatants. The freezer lyophilized $\left(80^{\circ} \mathrm{C}\right)$ filtrates were then dried and stored in amber coloured bottled for further examinations.

In vitro assays: Assays were performed to observe the cell density ${ }^{17}$, swarming motility ${ }^{18}$, protein $^{19}$, protease ${ }^{20}$, hemolysis $^{21}$, hemagglutination ${ }^{22}$, hydrophobicity ${ }^{23}$, biofilm inhibition ${ }^{24}$ along with cell dry weight, cell wet weight and $\mathrm{pH}$. Luria Bertani broth with different concentrations of Melia dubia root extracts such as 10, 20, 30, 40, $50 \mathrm{mg} / \mathrm{mL}$ were taken with a Luria Bertani media without supplementation of extract as the control. The tests were carried out in triplicates to ensure the support of statistical analysis. In order to discriminate between the antibiotic and Quorum quenching activity, two antibiotics ciprofloxacin and trimethoprim each $2 \mathrm{mg} / \mathrm{mL}$ were used as reference for the control. The influence of the extract on E. coli was observed at regular time intervals of 12 , 24, 48 and $72 \mathrm{~h}$.

GC-MS analysis: The ethanolic extract derived from the bark of the plant was fed to GC-MS for analysis in a PerkinElmer Clarus 500 with mass spectroscopy detector to identify the active principle(s) in it. The oven temperature of $50^{\circ} \mathrm{C}$ for $1 \mathrm{~min}$ at $10^{\circ} \mathrm{C} / \mathrm{min}$ to $150^{\circ} \mathrm{C}$ for $1 \mathrm{~min}$ at $8^{\circ} \mathrm{C} / \mathrm{min}$ to $250^{\circ} \mathrm{C} / \mathrm{min}$ at $15^{\circ} \mathrm{C} / \mathrm{min}$ to $300{ }^{\circ} \mathrm{C}$ for $3 \mathrm{~min} .1 \mu \mathrm{L}$ of sample completely dissolved in ethanol was injected with helium $(1 \mathrm{~mL} / \mathrm{min})$ as carrier gas and with an injection temperature of $280^{\circ} \mathrm{C}$ at mass spectral range set $40-450 \mathrm{amu}$. The identification of the compounds was made by comparing their mass spectrum with Nation Institute of Standard and Technology (NIST) mass spectral library.

\section{In silico studies}

Homology modeling of uropathogenic $E$. coli SdiA: The amino acid sequence of UPEC SdiA (Swisprot accession number: Q8FGM5) and the NMR solution structure coordinates of E. coli SdiA (PDB Code: $2 \mathrm{AVX}$ ) were loaded into the Modeller 9v8. The primary sequence of $E$. coli SdiA and UPEC SdiA were aligned carefully and checked to avoid deletions or insertions in the conserved regions. A series of the UPEC SdiA model (100 models) were constructed independently. There were no differences in the number and organization of the secondary structural elements and no significant main chain deviations among that of 100 models. The model with the best PDF Total energy, PDF Physical energy and DOPE function was selected and chosen for the further steriochemical quality checks and docking studies (Figs. 1 and 2).

Evaluation of the streochemical qualities of uropathogenic $E$. coli SdiA: The streochemical qualities of the UPEC SdiA were accessed by Ramachandran plot. $90.7 \%$ of the residues were in the favoured region, $7.3 \%$ in allowed region and only $1.9 \%$ were in the disfavoured region (Fig. 3). The residues in the disallowed regions are located far away from the residue in the ligand binding site (LBS). These results indicate the Phi and Psi backbone dihedral angles in the UPEC model are reasonably accurate. 


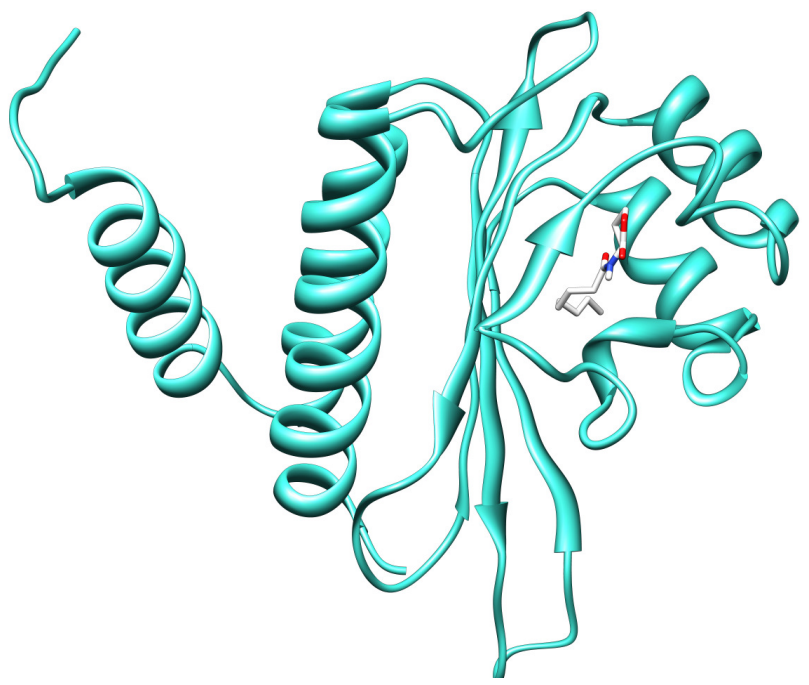

Fig. 1. A ribbon diagram showing the overall structure of the UPEC sdiA. The bound $\mathrm{C}_{8} \mathrm{HSL}$ molecular at active site is shown as stick

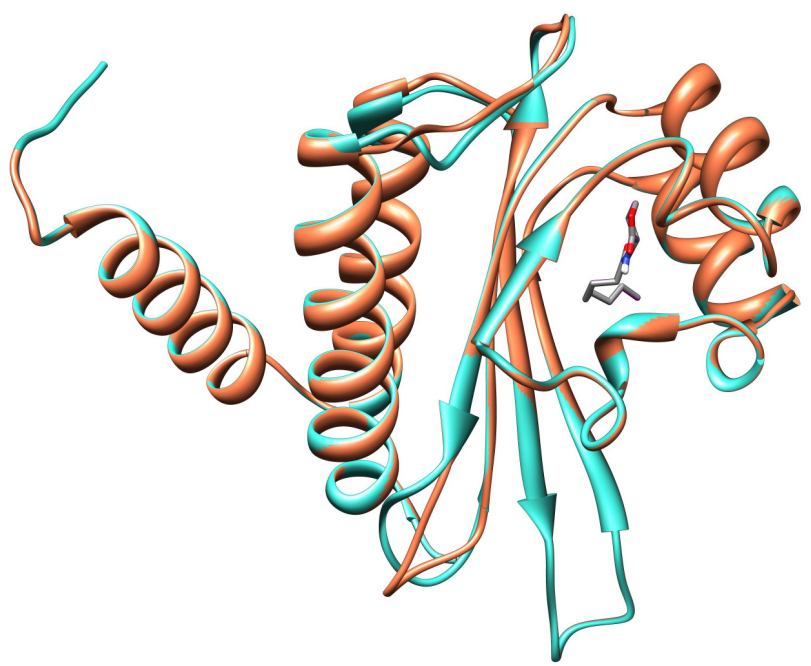

Fig. 2. Ribbon diagram of superposed E. coli sdiA (Coral) and UPEC sdiA (Turquoise)

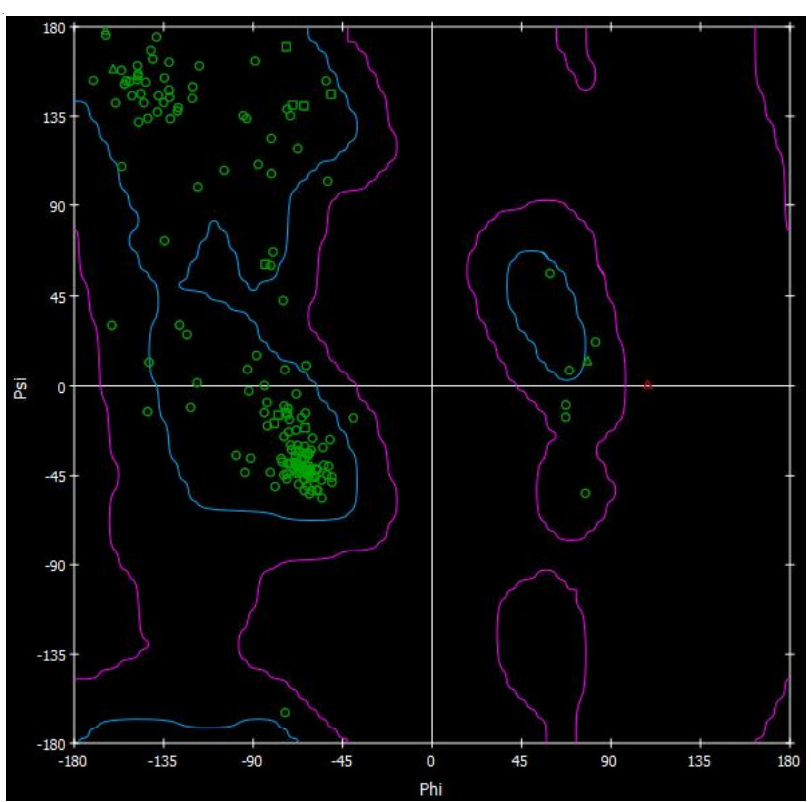

Fig. 3. Ramachandran plot of the final model obtained by PROCKECK
To access the quality of the model further, the Z-score was calculated using PROSA web server in order to check the overall model quality and to measure the deviation of the total energy of the structure with respect to an energy distribution derived from random confirmations. Fig. 4 shows the location of the Z-score for UPEC SdiA. The value -6.12 is in the range of native conformation. Hence the model was chosen for the further docking studies.

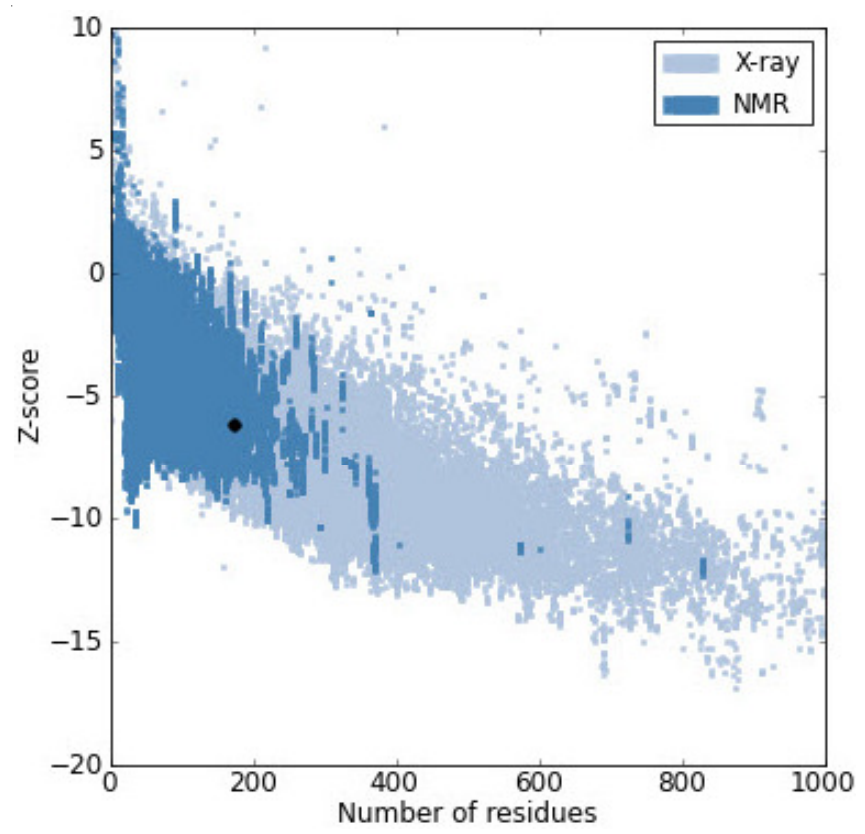

Fig. 4. Z-Plot of final model generated by ProSA-Web server

Ligand preparation: The 39 compounds reported by the GC-MS were drawn using the SymxDraw. The ligands files were prepared for docking using Schrodinger Liprep software. In addition to the generation of energy minimized 3D structure, Schrodinger Ligprep was also used for adding hydrogens. The main objective of using Ligprep was to obtain low energy 3D structure of the set of ligands to use in further computational studies. OPLS-2005 force field was utilized to optimize the geometry and minimize the energy.

Docking studies: Docking studies of the ligands were performed using the modeled UPEC SdiA structure. All the docking experiments were performed using the program Grid Based Ligand Docking with Energetics (GLIDE) module in Schrodinger. Coordinate of the modeled UPEC SdiA structure was prepared for Glide calculations by running the protein preparation wizard. Minimization were run until the average root mean square deviation (RMSD) of the non hydrogrn atom reached $0.290 \AA$. Glide uses two boxes that share a common centre to organize its calculations: A larger enclosing box and smaller binding box. The grids themselves or calculated within the space defined by the enclosing box. The binding box defines the space through which the centre of the defined ligand will be allowed to move during docking calculations. It provides a measure of the effective size of the search space. The only requirement on the enclosing box is that it be large enough to contain all ligand atoms, even when the ligand centre is place at the edge or vertex of the binding box. Grid files were generated using the $\mathrm{C}_{8} \mathrm{HSL}$ to the centre of the two boxes. The 
size of the binding box was set at $20 \AA$ in order two explore a large region of the protein. The compounds were subjected two flexible docking using the pre computed grid files. For each compound the 100 top score poses were saved and only the best scoring pose was analyzed.

Statistical analysis: The mean \pm SE of error was calculated and for the probability of differences below $5 \%$, the value was considered significant ${ }^{25}$.

\section{RESULTS AND DISCUSSION}

Extracts from the roots of Melia dubia was found to effectively suppress some of the major virulence characters of uropathogenic Escherichia coli like the formation of biofilms, hemolysin production, hydrophobicity, protease ctivity and swarming motility (Figs. 5-9). Hence the activity of ethanolic extract of the root is dealt in detail.

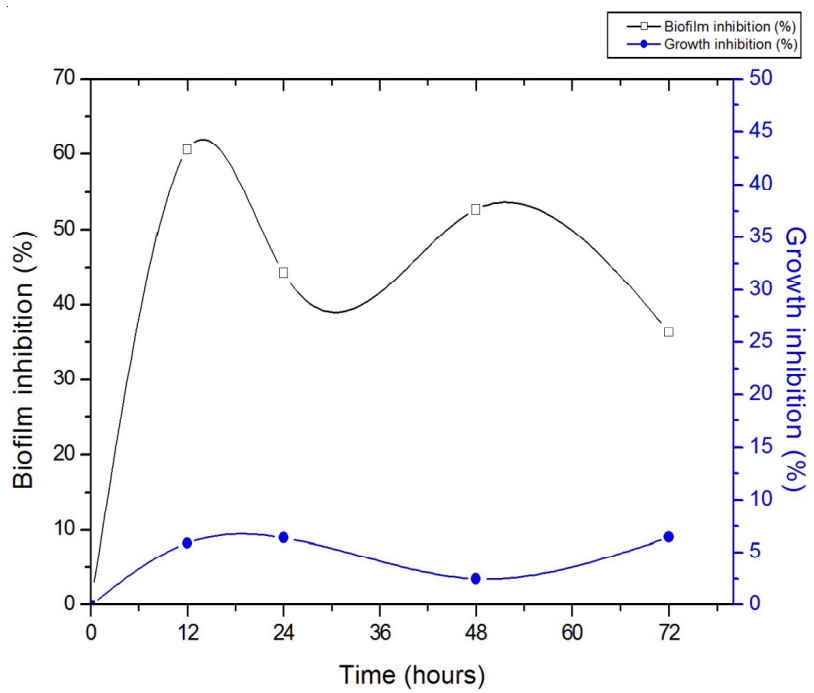

Fig. 5. Potential biofilm inhibiting efficacy of $M$. dubia root ethanolic extract $(30 \mathrm{mg} / \mathrm{mL})$ at different time intervals. Maximum inhibition activity $(60.6 \%)$ was recorded at after $12 \mathrm{~h}$

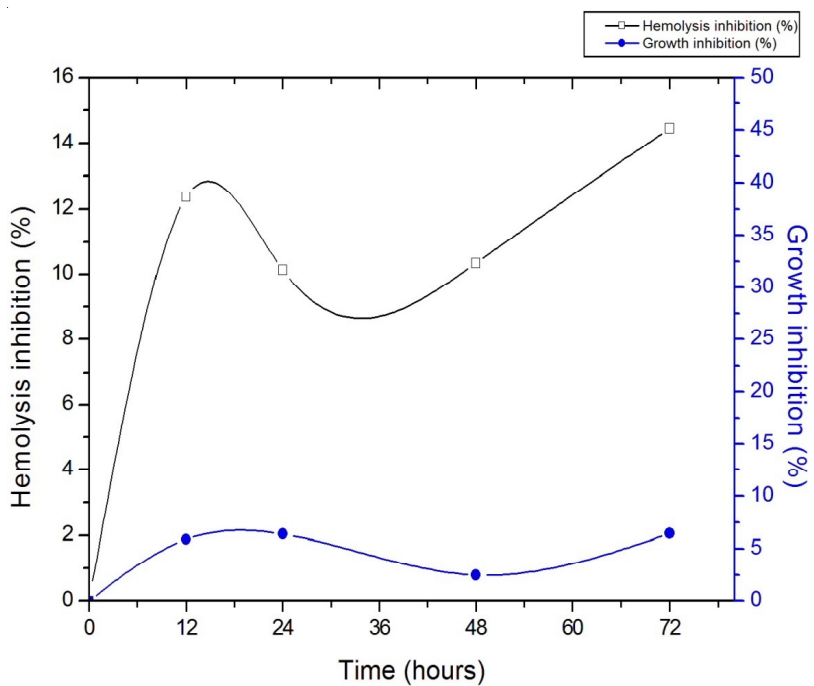

Fig. 6. Potential hemolysis inhibiting efficacy of $M$. dubia root ethanolic extract $(30 \mathrm{mg} / \mathrm{mL})$ at different time intervals. Maximum inhibition activity $(12.36 \%)$ was recorded at after $12 \mathrm{~h}$

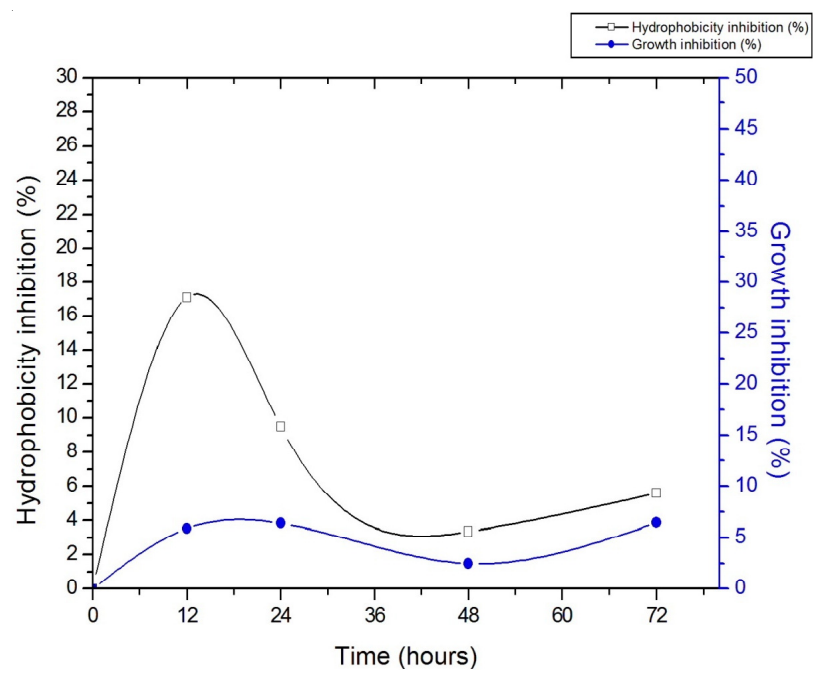

Fig. 7. Potential hydrophobicity inhibiting efficacy of $M$. dubia root ethanolic extract $(30 \mathrm{mg} / \mathrm{mL})$ at different time intervals. Maximum inhibition activity $(17.10 \%)$ was recorded at after $12 \mathrm{~h}$

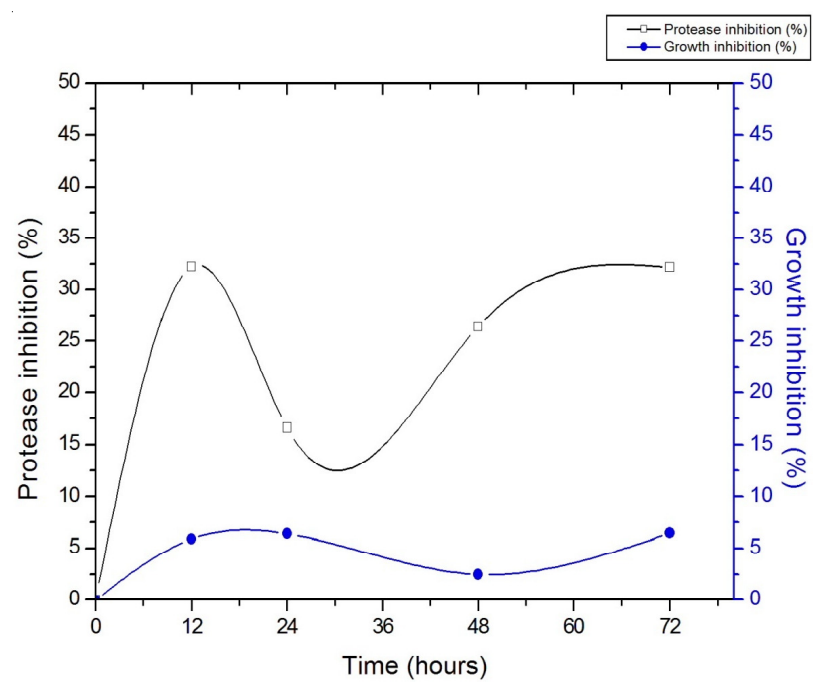

Fig. 8. Potential protease inhibiting efficacy of $M$. dubia root ethanolic extract $(30 \mathrm{mg} / \mathrm{mL})$ at different time intervals. Maximum inhibition activity $(32.24 \%)$ was recorded at after $12 \mathrm{~h}$

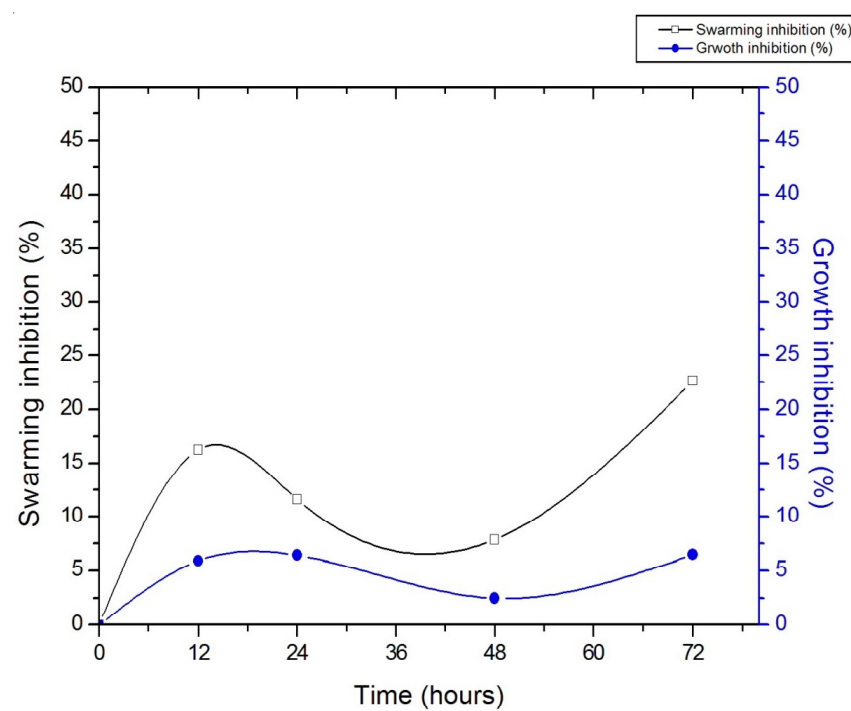

Fig. 9. Potential swarming inhibiting efficacy of $M$. dubia root ethanolic extract $(30 \mathrm{mg} / \mathrm{mL})$ at different time intervals. Maximum inhibition activity $(16.27 \%)$ was recorded at after $12 \mathrm{~h}$ 
In vitro assays: Bacteria that adhere to the surfaces, aggregate forming biofilms which resist the antibiotic action on the bacteria. Thus biofilm is considered to be one of the important virulence factors of $E$. coli to determine its multi resistance nature ${ }^{26}$. The use of boronic acids against Vibrio harveyi ${ }^{27}$ and coral-associated actinomycetes against Streptococcus pyogenes $^{28}$ are examples for attenuation of the biofilm to control the virulence of the bacteria. Here the root extracts of the plant Melia dubia is employed to quench the quorum sensing of E. coli and thereby controlling the expression of virulence characters in $E$. coli. The maximum inhibition of $60.6 \%$ was observed in $24 \mathrm{~h}$ when $30 \mathrm{mg} / \mathrm{mL}$ of the extract was used that coheres with our earlier studies ${ }^{29}$.

E. coli releases hemolytic toxins like RTX (repeat toxin) that causes the destruction of human blood cells in and around the urinary tract lysing for about $90 \%$ of granulates in $1 \mathrm{~h}^{30}$. In infancy the cytotoxicity can cause hemolytic anemia, thrombocytopenia and acute renal failure ${ }^{31}$. When the ethanolic root extract $(30 \mathrm{mg} / \mathrm{mL})$ of Melia dubia was treated against $E$. coli it showed an overall subjection of $c a .12 .36 \%$ in $24 \mathrm{~h}$ and the maximum being $14.45 \%$ for $72 \mathrm{~h}$.

E. coli exposed to the Melia dubia extract $(30 \mathrm{mg} / \mathrm{mL})$ showed a declined hydrophobic character of $\mathrm{ca} .17 .1 \%$ after $24 \mathrm{~h}$. Hydrophobicity of bacteria determines the efficiency of attachment of the bacteria over air-water interface or any other suitable substratum where their survival is made easy by the host environmental conditions, hence when the attachment is stronger the activity of the bacterium also is stronger. The pathogenic $E$. coli was found to possess a mannose resistant adhesion which is responsible for their attachment.

According to Nick and group, PicU is the second serine protease autotransporter of uropathogenic $E$. coli which contributes to the intimate adherence of $E$. coli towards mammalian cells. Commonly, proteases are enzymes that are responsible for the destruction of proteins as it introduces peptide bond breakages. In bacteria, these enzymes are useful in their safe and instant attachment over the cellular surfaces. The decline in the activity of these enzymes would possibly subjugate the colonization of bacteria and the biofilm formation, this was achieved by the Melia dubia extracts and the maximum was pragmatic after $24 \mathrm{~h}(32.24 \%)$.

The swarming motility of uropathogenic E. coli is through certain surfactants that enhance the movement by reducing the surface tension. The high swarming motility of bacteria may lead to increased biofilm formation due to their free movement thus increasing the rate of infection. Lowering the motility by interpreting their quorum sensing will provide a better way of inducing biofilm destruction. Here the technique was done using the extract of Melia dubia roots and the result obtained was the highest of $22.64 \%$ after $72 \mathrm{~h}$.

Though certain factors that are in capable of defending over antibiotics like cell weight, cell density remain unaffected, other pathogenic properties are silenced by the Melia dubia extract. Thus the ethanol extract derived from the roots of Melia dubia plant proved competent in quenching the quorum sensing of uropathogenic E. coli.

\section{In silico studies}

Identification and analysis of potential compounds: As a control study, the $\mathrm{C}_{8} \mathrm{HSL}$ was docked to the protein, an exercise which resulted in reproducing the NMR solution structure pose of the compound that yields -9.4 as the G score with $0.029 \AA$ RMSD. G Score is nothing but the total GLIDE score: Sum of XP terms (Lipophilic EvdW, PhobEn, PhobEnHB, PhobEL, PairHB, HBond, Eleactro, SiteMap, Phi Stack, Cat, CLBR, LowM, Penalties, HBPenal, PhobicPenal, RoatPNAL). If higher the contribution of XP term, the total GLIDE score will be more. The score computed for this reference compound was used as reference value for identifying the possible leads. All those compounds that exhibited interaction energies above this threshold or in other word indicated binding weaker than the reference compound where eliminated from the list for further analysis. From this docking studies, it is observed that the compound $\mathbf{7}$ and $\mathbf{2 7}$ are having better GScore of 11.5, 11.2 respectively than the native ligand $\mathrm{C}_{8} \mathrm{HSL}$. Compound 7 is forms three hydrogen strong bond interactions with the amino acid residues TRP67, ASP80 and SER43 (Fig. 10). And compound 27 forms two hydrogen bond interactions with TRP 67 and SER 43 (Fig. 11). From the previous experiment it is proved that TRP67 and TYR 71 are highly conserved and key residue for LuxR type proteins and SER 43 is a homologus residue of SdiA family. Since compound $\mathbf{7}$ and $\mathbf{2 7}$ were able to make strong hydrogen bond interaction with these key residues, these compounds could be a possible reason for the quorum quenching activity. Hence this compound can be further evaluated for the individual activities.

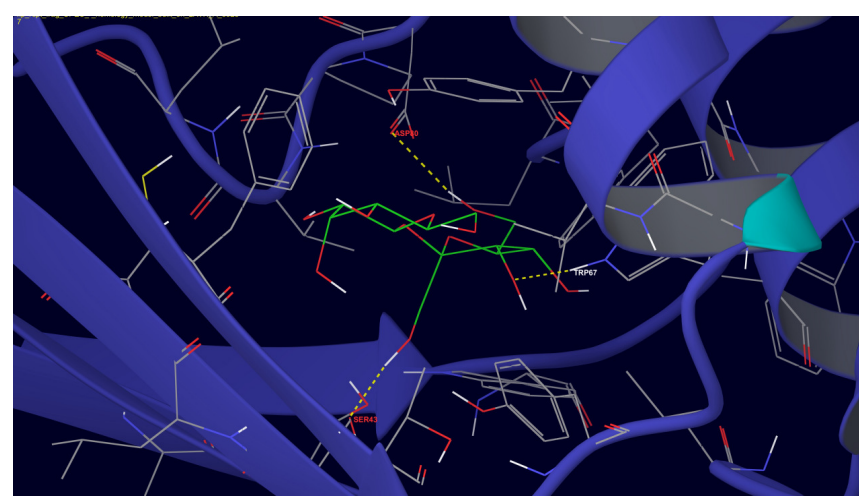

Fig. 10. Docking model of UPEC sdiA-C27. The hydrogen bond interactions with the key residues are show as dotted lines

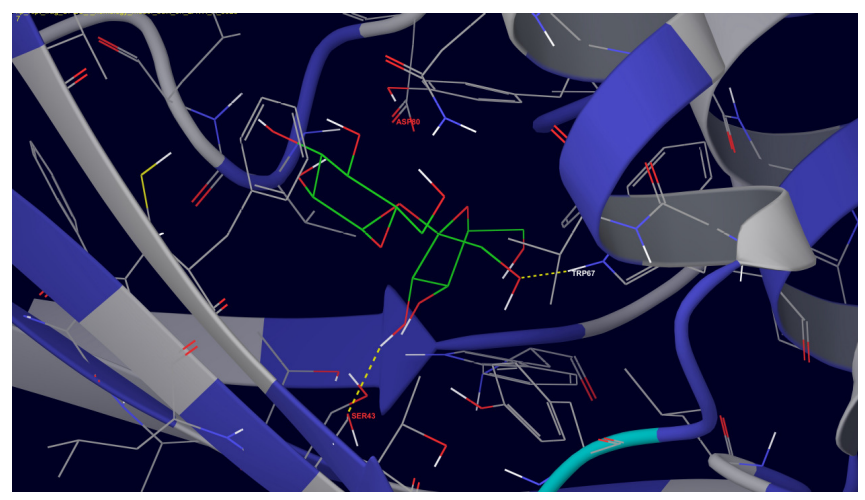

Fig. 11. Docking model of UPEC sdiA-C7. The hydrogen bond interactions with the key residues are show as dotted lines 


\section{Conclusion}

The potentiality of Melia dubia root in quenching the uropathogenic $E$. coli was proven from the overall analysis and its effeciency towards the virulence factors inhibition were analyzed. The in vitro and in silico analysis of the quorum quenching ligand present in Melia dubia active principle(s) gave promising results. The analysis also proved that the ligands present in Melia dubia can be raised as an efficient drug against the uropathogenic E. coli. The efficacy of the ligands in curbing uropathogenic $E$. coli will be focused further by in vitro and in vivo strategies.

\section{REFERENCES}

1. B. Foxman, Disease-a-Month, 49, 53 (2003).

2. $\quad$ L.E. Nicollece, Urol. Clin. North Am., 35, 1 (2008)

3. R.L. Vogt and L. Dippold, Public Health Rep., 120, 174 (2005).

4. J.N. Smith and B.M. Ahmer, J. Bacteriol., 185, 1357 (2003).

5. M.P. DeLisa, C.F. Wu, L. Wang, J.J. Valdes and W.E. Bentley, J. Bacteriol., 183, 5239 (2001).

6. S. Yang, C.R. Lopez and E.L. Zenchiedrich, Proc. Natl. Acad. Sci. (USA), 103, 2386 (2006)

7. Y. Yao, M.A. Martinez-Yamout and H.J. Dyson, J. Biomol. NHR, 31, 373 (2005).

8. S. Rahmati, S. Yang, A.L. Davidson and E.L. Zechietrich, Mol. Microbiol., 43, 677 (2002).

9. Y. Wei, A.C. Vollmer and R.A. LaRossa, J. Bacteriol., 183, 2259 (2001).

10. T.B. Rasmussen, T. Bjarnsholt, M.E. Skindersoe, M. Hentez, P. Krishofferson, M. Köte, J. Nielsen, L. Eberl and M. Givskov, J. Bacteriol., 187, 1799 (2004).

11. M. Hentzer and M. Givskov, J. Clin. Invest., 112, 1300 (2003).

12. G. Siromoney, D.G. Lal and C. Livingstone, Herbal Medicines of Narikoravas Folklore, Vol. 14, pp. 363-366 (1973).
13. T. Susheela, P. Balavani, J. Theeophilus, T.N. Reddy and P.U.M. Reddy, Curr. Sci., 94, 9 (2008).

14. P. Vijayan, C. Raghu, G. Ashok, S.A. Dhanaraj and B. Suresh, Indian J. Med. Res., 120, 24 (2004).

15. S. Malarvannan, R. Giridharan, S. Sekar, V.R. Prabavathy and S. Nair, J Biopestic., 2, 64 (2009).

16. V. Gupta, S. Saggu, R.K. Tulsawani, R.D. Sawhney and R. Kumar, Food Chem. Toxicol., 46, 1645 (2008).

17. D.M. Carlberg, Determining the Effects of Antibiotics on Bacterial Growth by Optical and Electrical Methods. In: Antibiotics in Laboratory Medicine, Williams \& Wilkins, Vol. 40, pp. 64-92 (1986).

18. J. Lee, T. Bansal, A. Jayaraman, W.E. Bentley and T.K. Wood, Appl. Environ. Microbiol., 73, 4100 (2003).

19. O.H. Lowry, N.J. Rosebrough, A.L. Farr and R.J. Randall, J. Biol. Chem., 193, 265 (1951).

20. M. Kunitz, J. Gen Physiol., 30, 291 (1947).

21. J.F. Van den Bosch, P. Postma, J. DeGraaff and D.M. MacLaren, FEMS Microbiol. Lett., 8, 75 (1980).

22. D.G. Evans, D.J. Evans and W. Joa, Infect. Immun., 18, 330 (1977).

23. J.S. Chapman and N.H. Georgopapadakou, Antimicrob. Agents Chemother, 32, 438 (1988).

24. O.M. Vandeputte, M. Kiendrebeogo, S. Rajaonson, B. Diallo, A. Mol, M.E. Jaziri and M. Baucher, Appl. Environ. Microbiol., 76, 243 (2010).

25. J.H. Zar, Biostatistical Analysis, Prentice Hall International, Inc. Press, London, edn. 4 (1988).

26. J.W. Costerton, P.S. Stewart and E.P. Greenberg, Science, 284, 1318 (1999).

27. N. Ni, G. Choudhary, M. Li, H.T. Chou, C.D. Lu, E.S. Gilbert and B. Wang, Chem. Biol. Drug Des., 74, 51 (2009).

28. P. Nithyanand, R. Thenmozhi, J. Rathna and S.K. Pandian, Curr. Microbiol., 60, 454 (2010).

29. V. Ravichandiran, K. Shanmugam, K. Anupama, S. Thomas and A. Princy, Eur. J. Med. Chem., 48, 200 (2012).

30. O.V. Gadeberg and I. Orskov, Infect. Immun., 45, 255 (1984).

31. D.A. Burke and A.T. Axon, Gut, 29, 41 (1988).

32. N.J. Parham, U. Srinivasan, M. Desvaux, B. Foxman, C.F. Marrs and I.R. Henderson, FEMS Microbiol. Lett., 230, 73 (2003). 